EPJ Web of Conferences 37, 04003 (2012)

DOI: $10.1051 /$ epjconf/20123704003

(C) Owned by the authors, published by EDP Sciences, 2012

\title{
On the Role of One Pion Exchange and Heavy Quark Spin Symmetry in Heavy Meson Molecules
}

\author{
M. Pavón Valderrama ${ }^{\mathrm{a}}$ and J.M. Nieves \\ Instituto de Física Corpuscular (IFIC), Centro Mixto CSIC-Universidad de Valencia, \\ Institutos de Investigación de Paterna, Apartado 22085, E-46071 Valencia, Spain
}

\begin{abstract}
In this contribution we consider the theoretical description of heavy mesonantimeson molecules from the effective field theory perspective. We are interested in the role of one pion exchange and heavy quark spin symmetry in the low energy description of the molecular states. We find that pion exchanges are weaker than naively expected. As a consequence, at lowest order in the effective expansion, the heavy meson-antimeson dynamics are driven by contact range interactions that are in turn heavily constrained by heavy quark spin symmetry. We find that if the $X(3872)$ is a $D \bar{D}^{*}$ bound state with quantum numbers $J^{P C}=1^{++}$, we should expect the existence of a $2^{++} D \bar{D}^{*}$ molecule with a mass of $4012 \mathrm{MeV}$. If we also assume the $X(3915)$ resonance to be molecular, we end up deriving the location of three new states with masses of 3710,3820 and $3855 \mathrm{MeV}$.
\end{abstract}

\section{Introduction}

The existence of heavy hadron molecules is to be expected from the observation that meson exchange forces can arise between heavy hadrons (as far as they contain a light quark) [1]. In analogy with the nuclear forces that bind the deuteron, the exchange forces among heavy hadrons might be able to induce binding as well. However, it is quite difficult to predict in advance the mass and quantum numbers of these molecular states. In this regard, the pioneering explorations of Ericson and Karl [2] and Törnqvist [3], in which they considered the exchange of the lightest meson (the pion), pointed out that heavy meson-antimeson bound states are more probable the heavier the hadrons, where the lightest system in which molecular states are expected to be formed is in the charm sector.

The discovery of the $X(3872)$ by the Belle Collaboration [4] probably represents so far the most promising candidate for a molecular state. With a mass $M=3871.68 \pm 0.17 \mathrm{MeV}$ [5], the closeness of the $X(3872)$ to the $D^{0} \bar{D}^{0 *}$ threshold is usually interpreted as a strong hint of its molecular nature. The quantum numbers of the $X(3872)$ are either $J^{P C}=1^{++}$or $2^{-+}[6,7]$ (see also Ref.[8] for a thorough discussion), from which the bound state picture is only compatible with the $1^{++}$assignment. Recently, a pair of new states have been observed in the bottom sector, the $Z_{b}(10610)$ and $Z_{b}(10650)$ [9], in the vicinity of the $B \bar{B}^{*}$ and $B^{*} \bar{B}^{*}$ thresholds respectively. They might also turn out to be molecular.

However, heavy meson molecules do not need to be as shallow as the $X(3872)$. It only happens that they are easier to identify if they are low-lying bound states. On the one hand, the $X(3872)$ contain a heavy quark-antiquark pair and is thus subjected to heavy quark symmetry. Therefore, the existence of symmetry partners of the $X(3872)$ will come as no surprise. On the other, the $X(3872)$ was just the first in a series of states above the open charm threshold, the so-called XYZ states, of which some might turn out to be molecular. In this contribution we will try to identify possible molecular partners of the $X(3872)$ among the XYZ states and predict the location of new states on the basis of heavy quark symmetry.

\footnotetext{
a e-mail: m.pavon.valderrama@ific.uv.es
}

This is an Open Access article distributed under the terms of the Creative Commons Attribution License 2.0, which permits unrestricted use, distribution, and reproduction in any medium, provided the original work is properly cited. 


\section{The Effective Field Theory Formulation of Molecular States}

Effective field theories (EFTs) are generic descriptions of low energy phenomena. They are grounded on the idea that low energy physics should be independent of the details of the (possibly unknown) high energy dynamics. This concept can be implemented by identifying the relevant symmetries and degrees of freedom at low energies and then writing down the most general lagrangian involving these ingredients. The approach is thus model-independent - no assumption is made about the nature of the high energy physics - but so far it also lacks predictive power as model-independence is achieved by including all possible physics. Predictability is restored with power counting, an ordering principle for sorting out the a priori infinite number of EFT interactions. Power counting identifies which interactions are more relevant at low energies and allows us to write any amplitude $\mathcal{A}$ as a power series in terms of the small expansion parameter $x_{0}$

$$
\mathcal{A}=\sum_{v \geq v_{0}} \mathcal{A}_{v} x_{0}^{v}
$$

where $x_{0}=Q / \Lambda_{0}$ is the ratio of the characteristic low energy scale $Q$ of the system over the high energy scale $\Lambda_{0}$. The advantage of power counting is clear: the EFT formulation is systematic, meaning that if we truncate the expansion of the amplitude at order $v_{\max }$ the error of the calculation is of order $x_{0}^{v_{\max }+1}$.

If we are interested in heavy meson molecules, the low energy degrees of freedom are the pion and the heavy meson and antimeson fields. The relevant symmetries are chiral symmetry, which constrains the pion exchanges, and heavy quark spin symmetry (HQSS) [10]. The characteristic low energy scale $Q$ is of the order of $m_{\pi}$, while the hard scale $\Lambda_{0} \sim 1 \mathrm{GeV}$ corresponds either to the momentum at which an external probe starts to resolve the compound nature of the heavy mesons or the chiral symmetry breaking scale. As we are dealing with heavy mesons, we can formulate a non-relativistic EFT in which the heavy meson-antimeson potential is a well-defined object that can be used as the building block of all computations (as happens in the Weinberg counting for nuclear forces [11]).

At lowest order (LO) in the power counting expansion the EFT potential naively consist of two contact interactions and one pion exchange (OPE). However, the presence of a bound state alters the counting and enhances the importance of the contact interactions at low energies [12,13]. Pions do not feel this enhancement and enter only at subleading orders. This means that at LO the EFT for heavy meson molecules is a purely contact range theory (basically equivalent to X-EFT [14]), representing a considerable simplification from the computational point of view.

We should stress that the conclusion that pions are subleading and hence perturbative is not so obvious as it seems. Naively we expect this to be true only up to center-of-mass momenta of $p \sim m_{\pi}$ or equivalently up to binding energies of $m_{\pi}^{2} / M_{P}$ ( $M_{P}$ being the mass of the heavy mesons), approximately $10 \mathrm{MeV}$ for hidden charm molecules. Above this energy the size of the OPE contribution is expected to become as big as the contact range piece, inducing a change in the counting. However, this is not necessarily the case. There are a series of techniques from atomic physics and nuclear EFT (see [15] and references therein) that allow us to determine the specific momentum for which the OPE potential starts to behave non-perturbatively. For heavy meson molecules the critical momentum is of the order of the hard scale in the charm sector, meaning that the EFT framework we advocate here is valid up to binding energies of $100 \mathrm{MeV}$ or more [16]. Only in the isoscalar bottom sector we find it necessary to treat OPE non-perturbatively, in which case we can apply the formalism of Ref. [17].

\section{Heavy Quark Spin Symmetry}

We can write the LO effective potential in momentum space as follows

$$
\begin{aligned}
V_{\mathrm{P} \overline{\mathrm{P}}}^{\mathrm{LO}}\left(\mathbf{q}, 0^{++}\right) & =C_{0 a}, \\
V_{\mathrm{P}^{*} \overline{\mathrm{P}} / \overline{\mathrm{P}}^{*}}^{\mathrm{LO}}\left(\mathbf{q}, 1^{+-}\right) & =C_{0 a}-C_{0 b}, \\
V_{\mathrm{P}^{*} \overline{\mathrm{P}} / \overline{\mathrm{P}}^{*}}^{\mathrm{LO}}\left(\mathbf{q}, 1^{++}\right) & =C_{0 a}+C_{0 b},
\end{aligned}
$$




\begin{tabular}{|c|c|c|c|c|}
\hline \hline$J^{P C}$ & $\mathrm{H} \overline{\mathrm{H}}$ & $E(\Lambda=0.5 \mathrm{GeV})$ & $E(\Lambda=1 \mathrm{GeV})$ & Exp (PDG [5]) \\
\hline $0^{++}$ & $D \bar{D}$ & $3706 \pm 10$ & $3712_{-17}^{+13}$ & - \\
\hline $1^{++}$ & $D^{*} \bar{D}$ & Input & Input & 3872 \\
$1^{+-}$ & $D^{*} \bar{D}$ & $3814 \pm 17$ & $3819_{-27}^{+24}$ & - \\
\hline $0^{++}$ & $D^{*} \bar{D}^{*}$ & Input & Input & 3917 \\
$1^{+-}$ & $D^{*} \bar{D}^{*}$ & $3953 \pm 17$ & $3956_{-28}^{+25}$ & 3942 \\
$2^{++}$ & $D^{*} \bar{D}^{*}$ & $4012 \pm 3$ & $4012_{-9}^{+4}$ & - \\
\hline \hline
\end{tabular}

Table 1. The HQSS molecular partners of the $X(3872)$ and $X(3915)$. The bound states are computed from the LO effective potential of Eqs. $(2-7)$, where the counterterms are fixed by reproducing the location of the $X(3872)$ and $X(3915)$ resonances. The calculations are performed for two values of the cut-off, and the error in the masses of the states are computed by assuming a $\Lambda_{\mathrm{QCD}} / m_{c}$ relative violation of HQSS in the countertems. Further details can be consulted in Ref. [18].

$$
\begin{aligned}
V_{\mathrm{P}^{*} \overline{\mathrm{P}}^{*}}^{\mathrm{LO}}\left(\mathbf{q}, 0^{++}\right) & =C_{0 a}-2 C_{0 b}, \\
V_{\mathrm{P}^{*} \overline{\mathrm{P}}^{*}}^{\mathrm{LO}}\left(\mathbf{q}, 1^{+-}\right) & =C_{0 a}-C_{0 b}, \\
V_{\mathrm{P}^{*} \overline{\mathrm{P}}^{*}}^{\mathrm{LO}}\left(\mathbf{q}, 2^{++}\right) & =C_{0 a}+C_{0 b},
\end{aligned}
$$

where the subscripts indicate the particle channel $\left(\mathrm{P} \overline{\mathrm{P}}, \overline{\mathrm{P}}^{*} / \mathrm{P}^{*} \overline{\mathrm{P}}, \mathrm{P}^{*} \overline{\mathrm{P}}^{*}\right)$ under consideration, with $P\left(P^{*}\right)$ the heavy pseudoscalar (vector) meson. It is worth commenting that at LO the particle channels are uncoupled: coupling effects enter at subleading orders only. The EFT potential is to be iterated in the Lippmann-Schwinger potential, $T=V+V G_{0} T$, where we search for poles of the T-matrix as they can be identified with the bound states of the system. However, the potential is singular at high momenta and requires a regularization procedure to make sense of it. For this, we have included a gaussian regulator with a momentum cut-off $\Lambda$. We vary the cut-off in the $0.5-1.0 \mathrm{GeV}$ window (that is, we take a cut-off of the order of the hard scale $\Lambda_{0}$ ) in order to check for the cut-off independence of the results. We notice that the check is a posteriori, as cut-off independence is already guaranteed from the renormalization group properties of the EFT framework. For a detailed description of the process we refer the reader to Ref. [18].

It is interesting to notice the strong constraints that HQSS imposes on the EFT potential. A remarkable consequence is the following

$$
\begin{aligned}
V_{\mathrm{P}^{*} \overline{\mathrm{P}} / \bar{P}_{\overline{\mathrm{P}}}^{*}}^{\mathrm{LO}}\left(\mathbf{q}, 1^{+-}\right) & =V_{P^{*} \bar{P}^{*}}^{\mathrm{LO}}\left(\mathbf{q}, 1^{+-}\right), \\
V_{\mathrm{P}^{*} \overline{\mathrm{P}} / \mathrm{P}^{*}}^{\mathrm{LO}}\left(\mathbf{q}, 1^{++}\right) & =V_{P^{*} \bar{P}^{*}}^{\mathrm{LO}}\left(\mathbf{q}, 2^{++}\right) .
\end{aligned}
$$

The first line explains the similar energies of the $Z_{b}(10610)$ and $Z_{b}(10650)$ resonances relative to the $B \bar{B}^{*}$ and $B^{*} \bar{B}^{*}$ thresholds (the quantum numbers of the $Z_{b}$ 's are believed to be $1^{+-}$), see also the more complete discussion of Refs. $[19,20]$. The second line indicates that if we have a $1^{++}$heavy mesonantimeson bound state, we should expect the existence of a $2^{++}$partner with a similar binding energy. This is relevant in the charm sector: if we accept the assumption that the $X(3872)$ is a $1^{++} D \bar{D}^{*}$ bound state, we can predict the existence of a $2^{++} D^{*} \bar{D}^{*}$ molecule with a mass of $4012 \mathrm{MeV}$, which we call the $X(4012)$. The prediction of this $X(4012)$ resonance is apparently robust: the bound state is still there even after considering the possible effects of HQSS and isospin violations, not to mention shifts in the binding energy induced by the OPE potential.

For predicting molecular partners of the $X(3872)$ beyond the $X(4012)$ we must identify an additional $X Y Z$ resonance as a heavy meson molecule. In this regard, two interesting candidates are the $X(3915)$ [21] and $X(3940)$ [22]. However, we may discard the second: even though the $X(3940)$ is known to decay into $D \bar{D}^{*}$, suggesting a $1^{+-} D^{*} \bar{D}^{*}$ molecule, the production mechanism for this resonance $\left(e^{+} e^{-} \rightarrow J / \Psi X(3940)\right)$ indicates that a positive C-parity assignment is more probable than a negative C-parity one. In contrast, the identification of the $X(3915)$ as a $0^{++} D^{*} \bar{D}^{*}$ bound state is free of problems and agrees with the (scarce) available data on this resonance [23]. From the $X(3915)$ we obtain a total of six molecular partners (four of them predictions), which can be consulted in Table 1. 
It is very interesting to notice that we predict a $1^{+-}$state at $3955 \mathrm{MeV}$, not far way from the $X(3940)$ region, which leads to the possibility of reconsidering the $X(3940)$ as a negative C-parity state and plausible molecular partner of the $X(3872)$. We also notice that some of the states of Table 1 have been predicted by other unrelated theoretical approaches [24-27]. As happened with the $X(4012)$ case, the results of Table 1 are robust with respect to the inclusion of subleading order effects, such as HQSS violations, the OPE potential, and the explicit inclusion of particle coupled channel dynamics [18].

To summarize, we have employed HQSS to predict the existence of molecular partners of the $X(3872)$ and $X(3915)$ within an EFT framework in which pions are perturbative. The application of the EFT formalism is very interesting in the sense that we can know in advance up to what extent we can trust the theory. Contrary to naive expectations, the effect of pion dynamics in the location of the bound states is small (a few MeV at most). However, the role of HQSS in the molecular spectrum can hardly be overstated. Of course, we do not forget that the EFT predictions also rely on the assumption that the $X(3872)$ and $X(3915)$ are molecular. This idea seems to be justified in the $X(3872)$, where there is certain evidence of its molecular nature, but in the case of the $X(3915)$ the identification is rather tentative. In this sense, while the existence of the $X(4012)$ is relatively well-grounded, the three states derived from the $X(3915)$ (i.e. the $X(3710), X(3820)$ and $X(3955)$ of Table 1$)$ should be granted a more conjectural status. Nonetheless, the agreement with other theoretical approaches plus the possible identification of the $X(3955)$ with the $X(3940)$ might indicate that we are on the right track.

This work was supported by the DGI under contract FIS2011-28853-C02-02, the Generalitat Valenciana contract PROMETEO/2009/0090, the Spanish Ingenio-Consolider 2010 Program CPAN (CSD2007-00042) and the EU Research Infrastructure Integrating Initiative HadronPhysics2.

\section{References}

1. M. B. Voloshin and L. B. Okun, JETP Lett. 23 (1976) 333

2. T. E. O. Ericson and G. Karl, Phys. Lett. B 309 (1993) 426.

3. N. A. Tornquist, Z. Phys. C 61 (1994) 525.

4. S. K. Choi et al. [Belle Collaboration], Phys. Rev. Lett. 91 (2003) 262001.

5. J. Beringer et al. [Particle Data Group Collaboration], Phys. Rev. D 86, 010001 (2012).

6. A. Abulencia et al. [CDF Collaboration], Phys. Rev. Lett. 98 (2007) 132002.

7. S. -K. Choi et al., Phys. Rev. D 84 (2011) 052004.

8. C. Hanhart et al., Phys. Rev. D 85 (2012) 011501.

9. A. Bondar et al. [Belle Collaboration], Phys. Rev. Lett. 108, 122001 (2012).

10. N. Isgur and M. B. Wise, Phys. Lett. B 232 (1989) 113; Phys. Lett. B 237 (1990) 527.

11. S. Weinberg, Phys. Lett. B 251, 288 (1990).

12. D. B. Kaplan, M. J. Savage and M. B. Wise, Phys. Lett. B 424 (1998) 390; Nucl. Phys. B 534 (1998) 329.

13. M. C. Birse, J. A. McGovern and K. G. Richardson, Phys. Lett. B 464 (1999) 169.

14. S. Fleming, M. Kusunoki, T. Mehen and U. van Kolck, Phys. Rev. D 76, 034006 (2007).

15. M. C. Birse, Phys. Rev. C 74 (2006) 014003.

16. M. Pavon Valderrama, Phys. Rev. D 85 (2012) 114037.

17. J. Nieves and M. Pavon Valderrama, Phys. Rev. D 84 (2011) 056015.

18. J. Nieves and M. Pavon Valderrama, arXiv:1204.2790 [hep-ph].

19. M. B. Voloshin, Phys. Rev. D 84 (2011) 031502.

20. T. Mehen and J. W. Powell, Phys. Rev. D 84 (2011) 114013.

21. S. Uehara et al. [Belle Collaboration], Phys. Rev. Lett. 104 (2010) 092001.

22. K. Abe et al. [Belle Collaboration], Phys. Rev. Lett. 98 (2007) 082001.

23. T. Branz, T. Gutsche and V. E. Lyubovitskij, Phys. Rev. D 80 (2009) 054019.

24. L. Maiani, F. Piccinini, A. D. Polosa and V. Riquer, Phys. Rev. D 71 (2005) 014028.

25. D. Gamermann, E. Oset, D. Strottman and M. J. Vicente Vacas, Phys. Rev. D 76 (2007) 074016.

26. D. Gamermann and E. Oset, Eur. Phys. J. A 33 (2007) 119.

27. R. Molina and E. Oset, Phys. Rev. D 80 (2009) 114013. 